\title{
A REMARK ON HARDY-TYPE INEQUALITY IN SPHERES
}

\section{SONGTING YIN and YU REN}

Department of Mathematics and Computer Science

Tongling University

Tongling, 244000 Anhui

P. R. China

e-mail: yst419@163.com

$$
\text { ry86901@163.com }
$$

\begin{abstract}
We obtain a Hardy type inequality with the best constant on the sphere and provide a simple and short proof. The result complements some inequalities in recent literatures.
\end{abstract}

\section{Introduction}

The classical Hardy inequality states that, for $n \geq 3$ and all $f \in C_{0}^{\infty}\left(\mathbb{R}^{n}\right)$,

$$
\int_{\mathbb{R}^{n}}|\nabla f|^{2} d x \geq \frac{(n-2)^{2}}{4} \int_{\mathbb{R}^{n}} \frac{f^{2}}{|x|^{2}} d x
$$

2010 Mathematics Subject Classification: Primary 26D10; Secondary 46E36.

Keywords and phrases: Hardy inequality, sphere, sharp constant.

This project is supported by TLXYXM (2018tlxyzd02, 2018tlxydxs154), DXSCXXM (2018 10383166), and NNSFC(11971253).

Received September 8, 2019

(ㄷ) 2019 Scientific Advances Publishers 
The constant $\frac{(n-2)^{2}}{4}$ is optimal and not attained in the Sobolev space $W^{1,2}\left(\mathbb{R}^{n}\right)$.

In [12], Kome and Özaydin obtained the improved Hardy inequalities in the Poincaré conformal disc model

$$
\int_{\mathbb{B}^{n}}|\nabla f|^{2} d V \geq \frac{(n-2)^{2}}{4} \int_{\mathbb{B}^{n}} \frac{f^{2}}{r^{2}} d V
$$

where $f \in C_{0}^{\infty}\left(\mathbb{B}^{n}\right) \quad$ and $\quad r=\log \frac{1+|x|}{1-|x|} \quad$ is the geodesic distance.

Furthermore, the constant $\frac{(n-2)^{2}}{4}$ is sharp.

There has been a lot of research concerning Hardy inequality in the Euclidean spaces and Riemannian manifolds due to its application to singular problems. We see [2], [3], [10], [11], [14] for the Euclid case and [4], [7], [8], [9], [12], [16] for the Riemannian case, and so on.

However, there are not many literatures discussing Hardy inequality on the sphere up to now. See [1], [5], [15], [17] and the references therein. Recently, Xiao ([15]) studied this issue and derived the following inequality:

$$
C_{1} \int_{\mathbb{S}^{n}} f^{2} d V+\int_{\mathbb{S}^{n}}|\nabla f|^{2} d V \geq \frac{(n-2)^{2}}{4}\left(\int_{\mathbb{S}^{n}} \frac{f^{2}}{d(p, x)^{2}} d V+\int_{\mathbb{S}^{n}} \frac{f^{2}}{(\pi-d(p, x))^{2}} d V\right) .
$$

Another Hardy-type inequality was obtained by the first author as follows ([17]):

$$
\frac{n-2}{2} \int_{\mathbb{S}^{n}} f^{2} d V+\int_{\mathbb{S}^{n}}|\nabla f|^{2} d V \geq \frac{(n-2)^{2}}{4} \int_{\mathbb{S}^{n}} \frac{f^{2}}{\tan ^{2} d(p, x)} d V .
$$

Here $d(p, x)$ is the geodesic distance from $p$ to $x$ on $\mathbb{S}^{n}$, and the constant $\frac{(n-2)^{2}}{4}$ is sharp. To see $L^{p}$-Hardy inequality on the sphere, we refer to [13]. 
In this short note, we will give a new Hardy-type inequality on the sphere with the best constant.

\section{The Main Result and its Proof}

Theorem 2.1. Let $\left(\mathbb{S}^{n}, g\right)(n \geq 3)$ be the $n$-sphere with sectional curvature 1. Then for any function $f \in C^{\infty}\left(\mathbb{S}^{n}\right)$, it holds

$\frac{n-2}{2} \int_{\mathbb{S}^{n}} \frac{f^{2}}{\tan ^{2} d(p, x)} d V \leq\left(\int_{\mathbb{S}^{n}} \frac{f^{2}}{\tan ^{2} d(p, x)} d V\right)^{\frac{1}{2}}\left(\int_{\mathbb{S}^{n}}|\nabla f|^{2} d V\right)^{\frac{1}{2}}+\frac{1}{2} \int_{\mathbb{S}^{n}} f^{2} d V$,

where $p$ is a fixed point in $\mathbb{S}^{n}$, and the constant $\frac{n-2}{2}$ is sharp.

Remark 2.2. Since on the sphere the Laplacian of the distance function is $\Delta d(p, x)=(n-1) \cot d(p, x)$, we let the inequality involve the term $\frac{f^{2}}{\tan ^{2} d(p, x)}$. So, it is a bit different in form from that in Euclidean spaces and some other type of Hardy inequalities. In Theorem 2.1, the second term in the right side of the inequality cannot be removed because it will lead to a contradiction if $f$ is a nonzero constant. To prove the result, we follow the method and idea from [6].

Proof. Let $f$ be a smooth function on $\mathbb{S}^{n}$ and $r(x)=d(p, x)$ denotes the distance function from the fixed point $p \in \mathbb{S}^{n}$. Then

$$
\begin{aligned}
\operatorname{div}\left(\frac{\nabla r}{\tan r}\right) & =\frac{\Delta r}{\tan r}+\nabla r\left(\tan ^{-1} r\right) \\
& =\frac{n-1}{\tan ^{2} r}-\frac{\sec ^{2} r}{\tan ^{2} r} \\
& =\frac{n-2}{\tan ^{2} r}-1,
\end{aligned}
$$


which together with divergence theorem yields

$$
\begin{aligned}
\int_{\mathbb{S}^{n}} \frac{f^{2}}{\tan ^{2} r} d V & =\frac{1}{n-2} \int_{\mathbb{S}^{n}} f^{2} \operatorname{div}\left(\frac{\nabla r}{\tan r}\right) d V+\frac{1}{n-2} \int_{\mathbb{S}^{n}} f^{2} d V \\
& =-\frac{1}{n-2} \int_{\mathbb{S}^{n}} \frac{\nabla r\left(f^{2}\right)}{\tan r} d V+\frac{1}{n-2} \int_{\mathbb{S}^{n}} f^{2} d V \\
& =-\frac{2}{n-2} \int_{\mathbb{S}^{n}} \frac{f\langle\nabla r, \nabla f\rangle}{\tan r} d V+\frac{1}{n-2} \int_{\mathbb{S}^{n}} f^{2} d V \\
& \leq \frac{2}{n-2} \int_{\mathbb{S}^{n}} \frac{f|\nabla f|}{|\tan r|} d V+\frac{1}{n-2} \int_{\mathbb{S}^{n}} f^{2} d V \\
& \leq \frac{2}{n-2}\left(\int_{\mathbb{S}^{n}} \frac{f^{2}}{\tan ^{2} r} d V\right)^{\frac{1}{2}}\left(\int_{\mathbb{S}^{n}}|\nabla f|^{2} d V\right)^{\frac{1}{2}}+\frac{1}{n-2} \int_{\mathbb{S}^{n}} f^{2} d V .
\end{aligned}
$$

It follows that

$$
\frac{n-2}{2} \int_{\mathbb{S}^{n}} \frac{f^{2}}{\tan ^{2} r} d V \leq\left(\int_{\mathbb{S}^{n}} \frac{f^{2}}{\tan ^{2} r} d V\right)^{\frac{1}{2}}\left(\int_{\mathbb{S}^{n}}|\nabla f|^{2} d V\right)^{\frac{1}{2}}+\frac{1}{2} \int_{\mathbb{S}^{n}} f^{2} d V
$$

In what follows, we show the constant $\frac{(n-2)}{2}$ is sharp.

Let

$$
f_{\delta}(x)=\frac{1}{|\tan r|^{\delta}}, \text { where } \delta=\frac{n-2}{2}
$$

Then $\left|\nabla f_{\delta}\right|=\frac{\delta \sec ^{2} r}{|\tan r|^{\delta+1}}$, and thus

$$
\int_{\mathbb{S}^{n}} \frac{f_{\delta}^{2}}{\tan ^{2} r} d V=\operatorname{Vol}\left(\mathbb{S}^{n-1}\right) \lim _{\epsilon \rightarrow 0} \int_{\epsilon}^{\pi-\epsilon} \frac{(\sin r)^{n-1}}{|\tan r|^{2 \delta+2}} d r
$$




$$
\begin{gathered}
\int_{\mathbb{S}^{n}}\left|\nabla f_{\delta}\right|^{2} d V=\operatorname{Vol}\left(\mathbb{S}^{n-1}\right) \lim _{\epsilon \rightarrow 0} \int_{\epsilon}^{\pi-\epsilon} \frac{\delta^{2} \sec ^{4} r(\sin r)^{n-1}}{|\tan r|^{2 \delta+2}} d r \\
\int_{\mathbb{S}^{n}} f_{\delta}^{2} d V=\operatorname{Vol}\left(\mathbb{S}^{n-1}\right) \lim _{\epsilon \rightarrow 0} \int_{\epsilon}^{\pi-\epsilon} \frac{(\sin r)^{n-1}}{|\tan r|^{2 \delta}} d r
\end{gathered}
$$

Since $f_{\delta}(x) \in C^{\infty}\left(\mathbb{S}^{n} \backslash\left\{p, r^{-1}(\pi)\right\}\right)$, and can be approximated by a family of smooth functions on the sphere $\mathbb{S}^{n}$, we compute

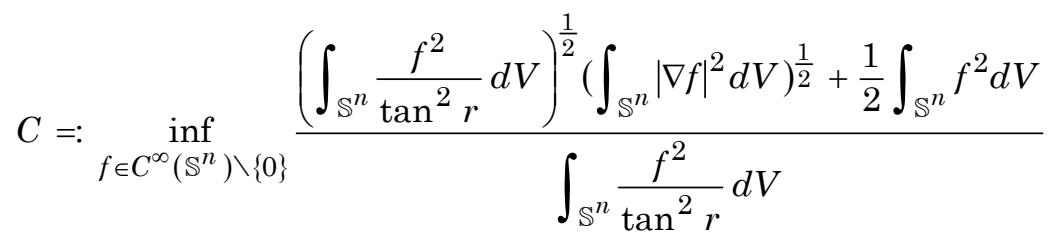

$$
\begin{aligned}
& \leq \frac{\left(\int_{\mathbb{S}^{n}} \frac{f_{\delta}^{2}}{\tan ^{2} r} d V\right)^{\frac{1}{2}}\left(\int_{\mathbb{S}^{n}}\left|\nabla f_{\delta}\right|^{2} d V\right)^{\frac{1}{2}}+\frac{1}{2} \int_{\mathbb{S}^{n}} f_{\delta}^{2} d V}{\int_{\mathbb{S}^{n}} \frac{f_{\delta}^{2}}{\tan ^{2} r} d V} \\
& =\frac{\left(\lim _{\epsilon \rightarrow 0} \int_{\epsilon}^{\pi-\epsilon} \frac{(\sin r)^{n-1}}{|\tan r|^{2 \delta+2}} d r\right)^{\frac{1}{2}}\left(\lim _{\epsilon \rightarrow 0} \int_{\epsilon}^{\pi-\epsilon} \frac{\delta^{2} \sec ^{4} r(\sin r)^{n-1}}{|\tan r|^{2 \delta+2}} d r\right)^{\frac{1}{2}}+\frac{1}{2} \lim _{\epsilon \rightarrow 0} \int_{\epsilon}^{\pi-\epsilon} \frac{(\sin r)^{n-1}}{|\tan r|^{2 \delta}} d r}{\lim _{\epsilon \rightarrow 0} \int_{\epsilon}^{\pi-\epsilon} \frac{(\sin r)^{n-1}}{|\tan r|^{2 \delta+2}} d r} \\
& =: I+I I \text {, }
\end{aligned}
$$

where, by L'Hospital rule,

$$
I=\left(\lim _{\epsilon \rightarrow 0} \frac{\int_{\epsilon}^{\pi-\epsilon} \frac{\delta^{2} \sec ^{4} r(\sin r)^{n-1}}{|\tan r|^{2 \delta+2}} d r}{\int_{\epsilon}^{\pi-\epsilon} \frac{(\sin r)^{n-1}}{|\tan r|^{2 \delta+2}} d r}\right)^{\frac{1}{2}}
$$




$$
\begin{aligned}
& =\left(\lim _{\varepsilon \rightarrow 0} \frac{\frac{\delta^{2} \sec ^{4} \varepsilon(\sin \varepsilon)^{n-1}}{|\tan \varepsilon|^{2 \delta+2}}}{\frac{(\sin \varepsilon)^{n-1}}{|\tan \varepsilon|^{2 \delta+2}}}\right)^{\frac{1}{2}} \\
& =\delta, \\
I I & =\frac{1}{2} \lim _{\epsilon \rightarrow 0} \frac{\int_{\epsilon}^{\pi-\epsilon} \frac{(\sin r)^{n-1}}{|\tan r|^{2 \delta}} d r}{\int_{\epsilon}^{\pi-\epsilon} \frac{(\sin r)^{n-1}}{|\tan r|^{2 \delta+2}} d r}=0 .
\end{aligned}
$$

This implies that

$$
C \leq \delta=\frac{n-2}{2}
$$

Thus the constant $\frac{n-2}{2}$ is sharp and the proof is complete.

In [18], the first author derived a Rellich type inequality on the sphere as follows:

$$
C(n) \int_{\mathbb{S}^{n}} \frac{f^{2}}{\sin ^{2} d(p, x)} d V+\int_{\mathbb{S}^{n}}|\nabla f|^{2} d V \geq \frac{n^{2}(n-4)^{2}}{16} \int_{\mathbb{S}^{n}} \frac{f^{2}}{\sin ^{4} d(p, x)} d V,
$$

where $C(n)=\frac{n(n-4)\left(n^{2}-2 n-4\right)}{8}$ and the constant $\frac{n^{2}(n-4)^{2}}{16}$ is sharp. One can also use the method above to obtain a similar result.

\section{References}

[1] A. Abolarinwa and T. Apata, $L^{p}$-Hardy-Rellich and uncertainty principle inequalities on the sphere, Advances in Operator Theory 3(4) (2018), 745-762.

DOI: https://doi.org/10.15352/aot.1712-1282

[2] P. Baras and J. A. Goldstein, The heat equation with a singular potential, Transactions of the American Mathematical Society 284(1) (1984), 121-139.

DOI: https://doi.org/10.1090/S0002-9947-1984-0742415-3 
[3] H. Brezis and J. L. Vázquez, Blow-up solutions of some nonlinear elliptic problems, Revista Matemática de la Universidad Complutense de Madrid 10(2) (1997), 443-469.

[4] G. Carron, Inégalités de Hardy sur les variétés Riemanniennes non-compactes, Journal de Mathématiques Pures et Appliquées 76(10) (1997), 883-891.

DOI: https://doi.org/10.1016/S0021-7824(97)89976-X

[5] F. Dai and Y. Xu, The Hardy-Rellich inequality and uncertainty principle on the sphere, Constructive Approximation 40(1) (2014), 141-171.

DOI: https://doi.org/10.1007/s00365-014-9235-5

[6] Y. Di, L. Jiang, S. Shen and Y. Jin, A note on a class of Hardy-Rellich type inequalities, Journal of Inequalities and Applications (2013); Article 84.

DOI: https://doi.org/10.1186/1029-242X-2013-84

[7] F. Du and J. Mao, Hardy and Rellich type inequalities on metric measure spaces, Journal of Mathematical Analysis and Applications 429(1) (2015), 354-365.

DOI: https://doi.org/10.1016/j.jmaa.2015.04.021

[8] L. D’Ambrosio and S. Dipierro, Hardy inequalities on Riemannian manifolds and applications, Annales de l'Institut Henri Poincare (C) Non Linear Analysis 31(3) (2014), 449-475.

DOI: https://doi.org/10.1016/j.anihpc.2013.04.004

[9] G. Grillo, Hardy and Rellich-type inequalities for metrics defined by vector fields, Potential Analysis 18(3) (2003), 187-217.

DOI: https://doi.org/10.1023/A:1020963702912

[10] J. P. Garcia Azorero and I. P. Alonso, Hardy inequalities and some critical elliptic and parabolic problems, Journal of Differential Equations 144(2) (1998), 441-476.

DOI: https://doi.org/10.1006/jdeq.1997.3375

[11] I. Kombe, The linear heat equation with highly oscillating potential, Proceedings of the American Mathematical Society 132(9) (2004), 2683-2691.

DOI: https://doi.org/10.1090/S0002-9939-04-07392-7

[12] I. Kombe and M. Özaydin, Improved Hardy and Rellich inequalities on Riemannian manifolds, Transactions of the American Mathematical Society 361(12) (2009), 6191-6203.

DOI: https://doi.org/10.1090/S0002-9947-09-04642-X

[13] X. Sun and F. Pan, Hardy type inequalities on the sphere, Journal of Inequalities and Applications (2017); Article 148.

DOI: https://doi.org/10.1186/s13660-017-1424-x 
[14] J. L. Vázquez and E. Zuazua, The Hardy inequality and the asymptotic behaviour of the heat equation with an inverse-square potential, Journal of Functional Analysis 173(1) (2000), 103-153.

DOI: https://doi.org/10.1006/jfan.1999.3556

[15] Y. Xiao, Some Hardy inequalities on the sphere, Journal of Mathematical Inequalities 10(3) (2016), 793-805.

DOI: https://doi.org/10.7153/jmi-10-64

[16] Q. Yang, D. Su and Y. Kong, Hardy inequalities on Riemannian manifolds with negative curvature, Communications in Contemporary Mathematics 16(2) (2014); Article 1350043, 24 pages.

DOI: https://doi.org/10.1142/S0219199713500430

[17] S. Yin, A sharp Hardy type inequality on the sphere, New York Journal of Mathematics 24 (2018), 1101-1110.

[18] S. Yin, A sharp Rellich inequality on the sphere, Mathematics 6(12) (2018); Article 288.

DOI: https://doi.org/10.3390/math6120288 